\title{
Cytological Features of Developing Anthers in Rose Balsam
}

\author{
Shujuan Yang ${ }^{1}$, Li Peng, and Han Bao \\ School of Life Science, Ningxia University, Helan Shan West Road No. 539, Xixia District, Yinchuan \\ 750021, Ningxia, P.R. China \\ Huiqiao Tian \\ School of Life Sciences, Xiamen University, Xiamen 361005, P.R. China
}

\begin{abstract}
ADDITIONAL INDEX WORDs. anther, Impatiens balsamina, lipids, polysaccharides, raphides, ultrastructure
Abstract. Our goal was to determine the ultrastructure features and the dynamic changes in polysaccharides and neutral lipids in developing anthers of rose balsam (Impatiens balsamina), and to better understand the mechanisms controlling male reproductive development. Transmission electron microscope (TEM) techniques were used to study the ultrastructural characteristics of the anthers, and histochemical methods were used to determine levels of polysaccharides and lipids. The cytokinesis in the microsporocyte meiosis was simultaneous type, and microspore tetrads were mainly tetrahedral. The pollen exine began to form at the tetrad stage. The mature pollen grains were oval-shaped and bicellular. Accumulation of reserve substances began at the late microsporogenesis stage, and an abundance of starch grains and lipids were stored in pollen grains at anthesis. Polysaccharides and lipids changed in different stages and played an important role in anther development. Moreover, the calcium oxalate crystals may protect the pollen and suggest that calcium distribution is related to anther development.
\end{abstract}

Impatiens balsamina is an annual herb, cultivated as a valued medicinal and ornamental plant all over China. It is also used as a popular dye, and in some places it is referred to as "henna." For centuries, the aboveground parts of this plant have been used in traditional Chinese medicine for their antimicrobial, antirheumatic, antipruritic, anti-inflammatory, antiallergic, and antitumoral properties, and for the treatment of difficult labor and puerperal pain (Kang et al., 2013; Shin et al., 2015). Many active ingredients have been isolated from I. balsamina, including phenolics, flavanols, anthocyanin pigments, and saponins (Oku and Ishiguro, 2002; Yang et al., 2001). Although metabolites from this plant have been extensively studied (Chua, 2016; Kang et al., 2013; Sakunphueak et al., 2013; Su et al., 2012), reports of detailed studies on anther development, cytological features, and distribution of nutritional reserves in the anthers of I. balsamina are scarce.

The flower, as an important reproductive organ, is believed to be a very complicated part of angiosperms. Specifically, the floral differentiation process and significant structural changes are complex mechanisms. Male reproductive development in higher plants is an intricate biological process that includes the formation of anthers with differentiated tissues, in which microspores and pollen are formed. As the anther morphology becomes established, the anther typically consists of microsporocytes at the center of each anther locule surrounded by four different cell layers: the epidermis, the endothecium, the middle layer, and the tapetum from the outer to the inner. The successful development of pollen grains within the anther

Received for publication 20 Nov. 2017. Accepted for publication 19 Jan. 2018. This work was supported by grants from the Ningxia Natural Science Foundation of China (No. NZ17033).

Electron microscopy was performed at the Xiamen University Instrument Center. The authors received assistance from many individuals during the course of the work and express their appreciation to all.

${ }^{1}$ Corresponding author. E-mail: nxyang11@126.com. requires active cooperation and interaction between the sporophytic and gametophytic molecules (Zhang et al., 2010).

Cell inclusions, especially calcium oxalate crystals are secondary metabolites and biominerals in plants, and are distributed among all taxonomic levels of angiosperms. Calcium oxalate crystals occur in five morphologically different forms, one or more of which are found in most angiosperm families (Franceschi and Horner, 1980; Franceschi and Nakata, 2005). Knowledge of plant crystals consists almost entirely of details of crystal structure, how the crystal forms within the cell, and distribution of crystals in mature organs. Crystal types, their specific distribution, and development are neglected aspects of crystallization. Many plants accumulate crystals of calcium oxalate, but how these crystals form remains unknown (Nakata, 2012, 2015). Since then, crystals of calcium and oxalate have been reported in more than 215 plant families (McNair, 1932).

In this study, the ultrastructure and histochemical changes associated with the various developmental stages of anthers were investigated, with a focus on changes of polysaccharides and lipids, which are nutritional materials. Study on the synthesis and distribution of nutritional constituents in the anthers can provide understanding viability and its role in reproductive fitness. In addition, the features of calcium oxalate crystals in the anthers, representing a nonmolecular synapomorphy were examined to gain insights into the reproductive biology and evolution of I. balsamina.

\section{Materials and Methods}

Plant Materials. Impatiens balsamina plants were grown on the campus of Xiamen University in Xiamen, China. Fresh anthers at different developmental stages [microspore mother cell (MMC), tetrads, microspore, bicellular pollen, and mature pollen] were collected and observed under a microscope to determine the exact stages. The characteristics of MMCs are loosening of the arrangement and surrounded by a thick callose 
wall; tetrads containing four microspores are also surrounded by a thick callose wall; microspore stage has a centrally located nucleus; bicellular pollen has two clear nuclei; mature pollen produces much nutritional materials.

SAMPle PREPARATION. A minimum of 20 anthers from each stage were collected from multiple flower buds and fixed with $2.5 \%(\mathrm{v} / \mathrm{v})$ glutaraldehyde in $0.1 \mathrm{M}$ phosphate buffer ( $\mathrm{pH} 7.0$ ) for $5 \mathrm{~h}$ at $4^{\circ} \mathrm{C}$. After rinsing with phosphate buffer three times for $30 \mathrm{~min}$, the samples were postfixed for $16 \mathrm{~h}$ in $1 \%(\mathrm{w} / \mathrm{v}) \mathrm{OsO}_{4}$ and maintained at $4{ }^{\circ} \mathrm{C}$. After three 30 -min rinses, the samples were dehydrated using an acetone series from $10 \%$ to $100 \%$ ( $10 \%$ to $90 \%$ acetone, $20 \mathrm{~min}$ each, and 100\% acetone, three times, $30 \mathrm{~min}$ each), and embedded in epoxy resin (SPIPon 812; Structure Probe, West Chester, PA).

E LE C T R ON M I C R O S COP E OBSERVATION. Embedded anthers were sliced into ultrathin $(60 \mathrm{~nm})$ sections with an ultramicrotome (Ultracut R 705930; Leica Microsystems, Wien, Austria). Ultrathin sections were placed on grids and stained with $2 \%$ uranyl acetate (Merck, Darmstadt, Germany) for $30 \mathrm{~min}$ and double stained with saturated lead citrate for $15 \mathrm{~min}$, followed by washing and drying. Images were obtained with a transmission electron microscope (JEM2100; JEOL, Tokyo, Japan).

LIGHT MICROSCOPE OBSERVATION. Embedded anthers were sliced into semithin $(1 \mathrm{~mm})$ sections and were placed onto slides. Staining was performed as described previously (Hu, 1990). Thin sections were stained with $0.5 \%(\mathrm{w} / \mathrm{v})$ basic fuchsine dye (85734-3; Sigma-Aldrich, St. Louis, MO) based on the periodic acid-Schiff reaction for $30 \mathrm{~min}$ at room temperature. The rationale locating polysaccharides and starch is that periodate can cleave glycol sites on (1-4)-linked glucose residues of polysaccharide analogues where basic fuchsin combines to display red color (Dahlqvist et al., 1965). These sections were then counter-stained with $0.3 \%(\mathrm{w} / \mathrm{v})$ Sudan Black B (19966-4; Sigma-Aldrich) for 30 min at $60{ }^{\circ} \mathrm{C}$ to detect neutral lipid droplets. The Sudan Black B has
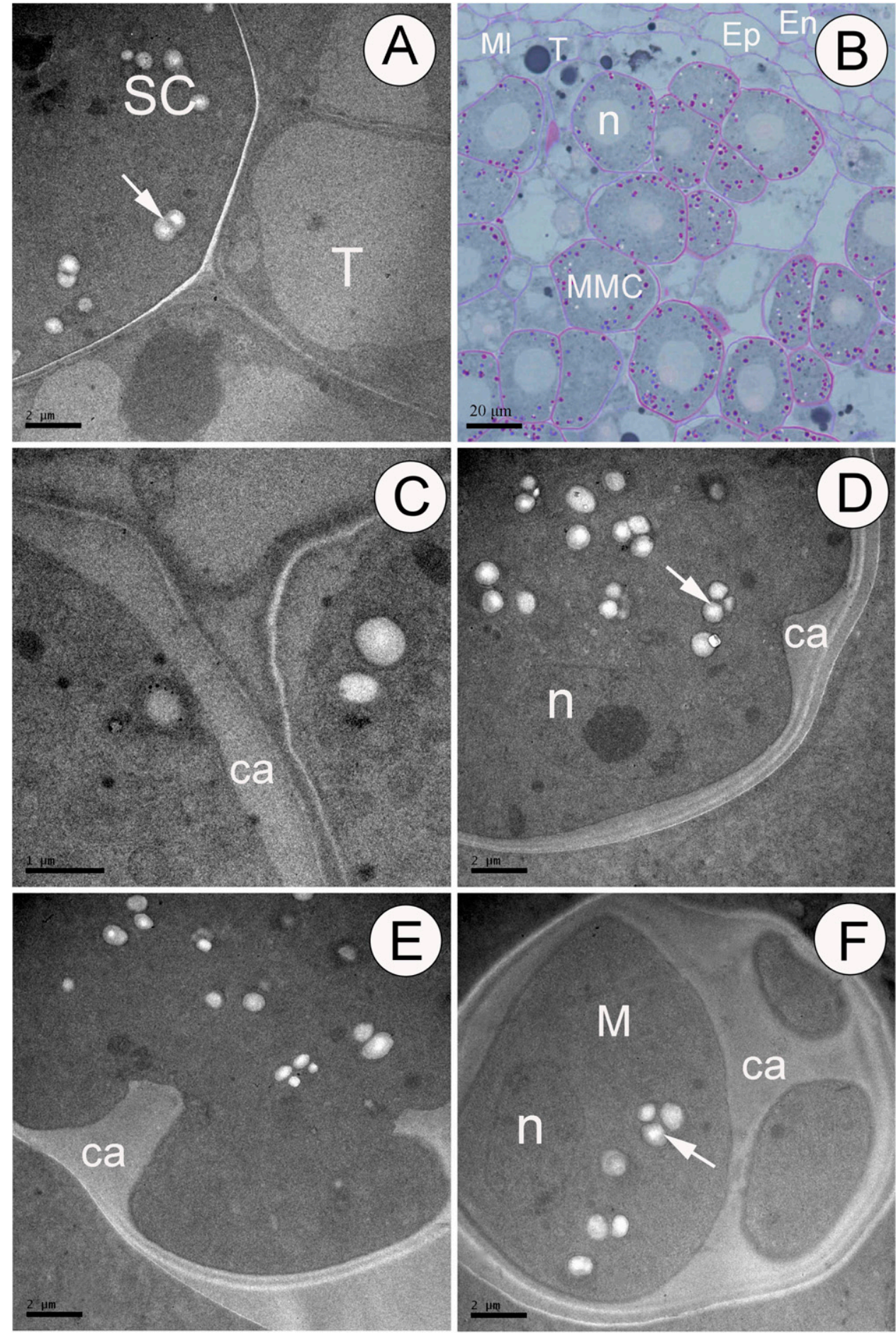

Fig. 1. Ultrastructure and starch and lipid distribution in young (Impatiens balsamina) anthers. (A) Transverse section of one anther at the sporogenous cell stage. Some small starch grains (arrow) are obvious in sporogenous cells, and there is a big vacuole in each tapetal cell, which are arranged closely. (B) Some round starch grains (stained red) are distributed in the cytoplasm of microspore mother cells (MMCs), which are also wrapped in red cell walls, some lipids (stained black) are present in tapetal cells, and there are also some degraded MMCs. (C) Cell wall of MMCs began to accumulate callose. (D) After meiosis I, the MMCs produced free nuclei, which were near the cell walls. With the sagging of cell walls in some areas, there were still some starch grains (arrow) in the cell. (E) Cell wall sags more heavily because of callose. (F) After meiosis II, a tetrad was formed and some bright starch grains remained (arrow). $\mathrm{SC}=$ sporogenous cell; $\mathrm{T}=$ tapetum; $\mathrm{En}=$ endothecium; Ep = epidermis; $\mathrm{ca}=$ callose; $\mathrm{M}=$ microspore; $\mathrm{Ml}=$ middle layer; $\mathrm{n}=$ nucleus. Bars: $(\mathbf{A}, \mathbf{C}, \mathbf{D}, \mathbf{E}$, and $\mathbf{F}) 2 \mu \mathrm{m},(\mathbf{B}) 20 \mu \mathrm{m}$. 
a special affinity with neutral lipid, which displayed black (Casselman, 1954). All sections were observed and photographed using a research microscope (DMR; Leica, Deerfield, IL).

\section{Results}

The anther of I. balsamina is capsule shaped. We observed four microsporangia in the transverse sections, and classified the pollen development process to five stages based on morphological characteristics: 1) MMC, 2) tetrad, 3) microspores, 4) bicellular pollen, and 5) mature pollen. Impatiens balsamina pollen grain belongs to bicellular type consisting of a vegetative cell and a generative cell.

Characteristics of the MMCs. The sporogenous cells were polygonal in shape (Fig. 1A) and arranged closely with the surrounding cells. The organelles were not mature, except for some white starch grains were seen in the cell. Sporogenous cells and tapetal cells were easily distinguished because the latter displayed higher vacuolization (Fig. 1A). The sporogenous cells then developed into MMCs, and large intercellular spaces were seen between MMCs (Fig. 1B). Histochemical staining and examination showed that cell walls and starch grains were red. A large and conspicuous nucleus was located in the center of the MMCs. A few large black lipid droplets were observed in some tapetal cells (Fig. 1B). TEM observations revealed that callose deposition was initiated within the primary walls of the MMCs (Fig. 1C).

Characteristics of The tetrads. During meiosis, MMCs produce four free nuclei that arranges tetrahedrally in the cell, and we can only find one of the four free nuclei because of the high magnification (Fig. 1D). As the cell wall begins to sag, starch grains were visible in the microspore cytoplasm (Fig. 1D). With the cell wall sagging very heavily, the MMC finally separated into four small cells, enclosed fully by a callose wall (Fig. 1E and F). We noticed that cytokinesis of $I$. balsamina was simultaneous and the arrangement of microspores in the tetrad was tetrahedral. The four microspores surrounded by the callose
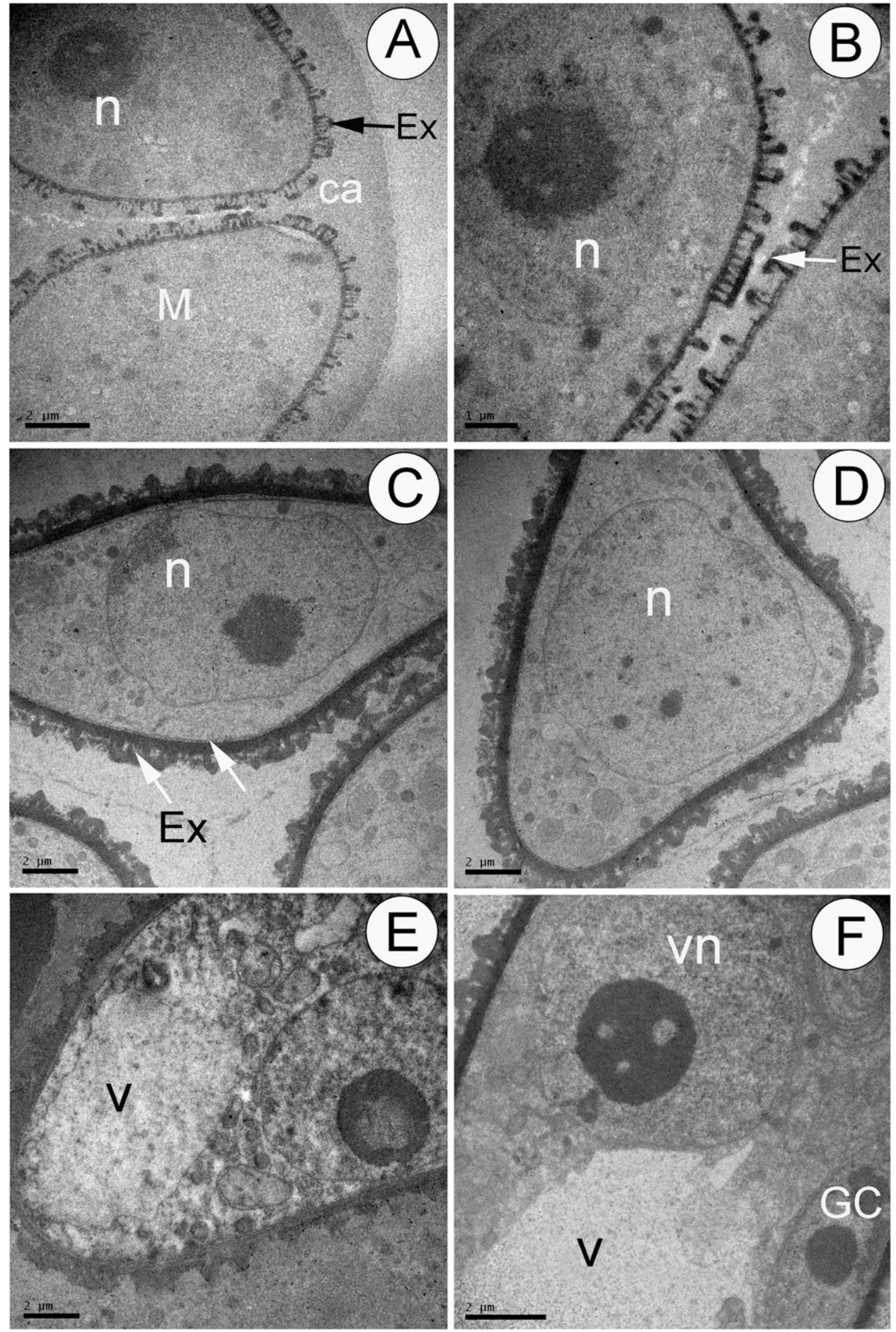

Fig. 2. Ultrastructure in young Impatiens balsamina anthers. (A) Microspore became bigger and the exine was produced, but tetrad was still surrounded by the callose wall. (B) After magnification, the exine showed interruptions and only had columella, foot layer, and endexine, but no tectum at this period. (C) The tectum grew thicker and the intine began to form, many organelles appeared. The nucleus was nearly round, and lay in the center of microspores. (D) The early microspores released from the tetrad were irregularly shaped but still had a large cell nucleus. (E) A big vacuole was formed and the cell nucleus and cytoplasm, rich in organelles, were pushed to the edge of the cell. (F) After mitosis, the microspore produced a large vegetative nucleus and a small generative cell, which is the bicellular pollen. The original generative cell that is semispherical clung to the pollen wall; the vegetative nucleus is free in the cytoplasm of pollen. $\mathrm{M}=$ microspore; $\mathrm{ca}=$ callose; $\mathrm{GC}=$ generative cell; v = vacuole; vn = vegetative nucleus; Ex = exin; $\mathrm{n}=$ nucleus. Bars: $(\mathbf{A}, \mathbf{C}, \mathbf{D}, \mathbf{E}$, and F) $2 \mu \mathrm{m},(\mathbf{B}) 1 \mu \mathrm{m}$. 
wall expanded and differentiated gradually, especially as the outer wall of the pollen began to appear (Fig. 2A). After enlargement, we found that the outer pollen wall was discontinuous and only had the columella, foot layer, and endexine, but no tectum at this stage (Fig. 2B). Some special substances deposited on the surface of pollen wall and developed into thicker tectum. The pollen intine also began to form with additional organelles appearing. The big round nucleus lies in the center of the microspore (Fig. 2C). This is the later stage of tetrad development, after which the pollen wall formation starts. Exine formation was basically complete by this late stage of microsporogenesis.

C H A R A C T E R I S T I C S O F MICROSPORE. After the degradation of tetrad callose wall, the four microspores are released from the tetrad. The early microspores with irregular shape still contain a large nucleus located in the center of the cell (Fig. 2D). During the late microspore development stage, one of the main characteristics of the microspore is that the nucleus position changes and cytoplasm is reorganized. At the same time, some small vacuoles appear in the cytoplasm of the microspore that eventually became a single large vacuole, which occupies almost the entire cell. The cell nucleus and cytoplasm are rich in organelles that are now pushed to the edge of the cell (Fig. 2E). As mentioned earlier, the large vacuole formation being the most obvious feature of microspore development, starts pushing the microspore nucleus to the cell periphery to create cell polarity. Hence, this stage is also known as the vacuolated microspore stage. At first, there are many small vacuoles, which gradually merge to form a large vacuole. Vacuolization is accompanied by the displacement of the microspore nucleus to an eccentric position against the microspore wall.

CHARACTERISTICS OF THE bicellular pollen. Microspore continues development with polarity for an unequal mitosis, which results in the formation of two unequal cells, a large vegetative cell, and a small generative cell, each containing a haploid nucleus. The
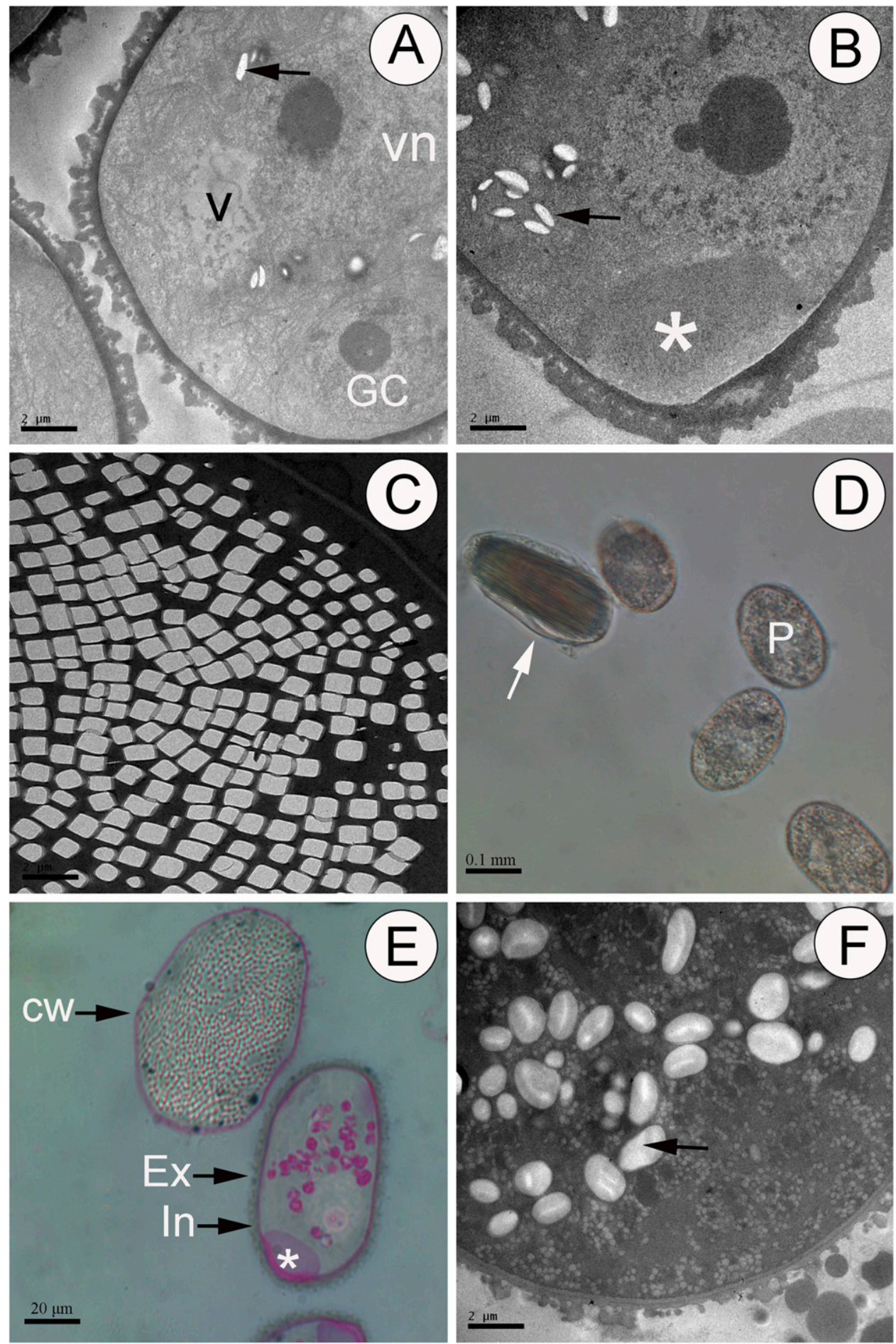

Fig. 3. Ultrastructure, and starch and lipid distribution in older Impatiens balsam anthers. (A) Large vacuole in vegetative cells became smaller and in generative cells grew rounder and bigger. Many endoplasmic reticula around the reproductive cells and a few oval starch grains appeared in pollen. (B) There were more oval starch grains (arrow) and a special structure (star) appeared. (C) Crystal cells were found in the mature anther of $I$. balsamina, transversal section of the ultrastructure showed that it was square shaped. (D) Examination using anther squash showed the crystal cell (arrow) was full of crystals arranged in bundles and the normal mature pollen was long oval, and full of nutritional material. (E) Both the cell wall and crystal were stained red in the crystal cell, and in the normal pollen the cell nucleus, apertures (star), and starch grains all stained red. (F) Abundant starch grains were stored in the mature pollen. $\mathrm{GC}=$ generative cell; $\mathrm{v}=$ vacuole; $\mathrm{vn}=$ vegetative nucleus; $\mathrm{Ex}=$ exin; $\mathrm{In}=$ intine; $\mathrm{cw}=$ cell wall; $\mathrm{P}=$ pollen. Bars: $(\mathbf{A}, \mathbf{B}, \mathbf{C}$, and $\mathbf{F}) 2 \mu \mathrm{m},(\mathbf{B}) 0.1 \mathrm{~mm},(\mathbf{E}) 20 \mu \mathrm{m}$. 
original generative cell is attached to the pollen wall, whereas the vegetative nucleus is free in the pollen cytoplasm (Fig. 2F). The obvious change seen early in the development of the bicellular pollen is that the large vacuole in the vegetative cell begins to disintegrate at this stage. The large vacuole in the vegetative cell breaks down into several smaller units and gradually disappears, and there is a small vacuole in the pollen (Fig. 3A). As the vacuoles disappear, the generative cells gradually detach from the pollen wall and migrates to the center of the cytoplasm to settle beside the vegetative nucleus. During the bicellular pollen stage, the starch grains are synthesized in the pollen after the large vacuole of the vegetative cell disappears (Fig. 3B).

Characteristics of THE MATURe pollen. In the mature anther of I. balsamina, we observed some crystal cells. Ultrastructure analysis of a transversal section revealed it is square shaped (Fig. 3C). When mature anthers were squashed on glass slides and observed under a microscope, we found some pollen grains were filled with crystals arranged in bundles, and the normal mature pollen grains had abundant nutritional particles (Fig. 3D). Histochemical analysis showed the pollen wall and the crystal were both dyed red in the crystal cell. In normal pollen, the cell nucleus, pollen wall, apertures, and starch grains were all dyed red (Fig. 3E). After the pollen matured, the vacuole disappeared completely, and subsequently, a large amount of storage material appeared in the pollen cytoplasm. Electron density also increased significantly in the cytoplasm, and it is difficult to distinguish the vegetative cell from the generative cell. The pollen wall has been completely formed. A mature pollen grain includes two cells - a vegetative cell and a generative cell. In mature pollen, there are no lipids, but a few starch grains. However, the pollen grains are at prebloom, preparing for pollination stage, and the lipid and starch content are both significantly increased (Fig. 3F).

\section{Discussion}

The anther structure of angiosperms is very complex and many detail developmental features are unclear. Our previous study showed that the anther wall of I. balsamina consists of four layers: epidermis, endothecium, middle layer, and tapetum. The development of the anther wall follows the basic type (Yang et al., 2013a, 2013b). In this study, the ultrastructural results showed that the MMCs gave rise to tetrahedral tetrads by meiosis. The cytokinesis in the microsporocyte meiosis was simultaneous: cell wall formation originated at the microsporocyte surface between the two nuclei, where the new walls grew centripetally and separated the MMC cytoplasm. The tetrad microspores were arranged tetrahedrally. The pollen exine began to form at the tetrad stage. We also found there were special apertures at the end of the pollen, which revealed polysaccharides and starch by histochemical methods. To our knowledge, this is the first report describing anther ultrastructure features in I. balsamina.

The metabolic pattern and distribution of starch grains and lipids varies in anthers of different plants. This is usually to ensure sufficient energy and material for pollen germination; mature pollen accumulates abundant nutrients in the form of starch grains or lipids (Pacini, 1996). In this study, we observed that the starch grains and lipids were abundant in the mature pollen grains of I. balsamina. Histochemical analysis of the anthers revealed there were a few smaller starch grains accumulated before the tetrad stage. After this stage, no starch grains were observed; however, starch grains reappeared at the late stage of bicellular pollen formation; notably, excessive amounts of starch grains and lipids were stored in mature pollen at anthesis. The result presented in this study has expanded our knowledge of the anther biology of this plant, and provided crucial insights into the anther development processes, including the late microsporogenesis stage, late bicellular pollen stage, and anthesis. Importantly, we confirmed that the accumulation of reserve substances began at the late microsporogenesis stage, and abundant amounts of starch grains and lipids were stored in pollen grains at anthesis.

Stem morphology and characteristics of calcium oxalate crystals in seven species within the genus Impatiens have been studied. The results indicated that there were little anatomical differences between the seven species in the genus Impatiens, but the diversity of the characteristics of calcium oxalate crystals was large (Lersten and Horner, 2005). According to morphological characters of the raphides, there are three types: focal raphides, clustered raphides, and scattered raphides. In addition, the shape, length, distribution, and the degree of abundance are different among the seven species. The characteristic of calcium oxalate crystals seems to be of some significance in Impatiens taxa. Oxalate crystals are very common in angiosperms, but few descriptions of them in pollen exist. Calcium oxalate crystals in Impatiens taxa have been described only superficially (Liu et al., 2006), and most references have merely mentioned that calcium oxalate crystals occurred in the leaf, stem, root, or seeds. We first observed the calcium oxalate crystals in the pollen grains by TEM. Thus, we studied the shape of the calcium oxalate crystals, their arrangement, and distribution, and surprisingly, we found that the transversal section of the calcium oxalate crystal revealed an almost square- or rectangle-shaped area.

\section{Conclusions}

This study provides basic information on male reproductive aspects of I. balsamina and sheds light on male developmental features in this species.

\section{Literature Cited}

Casselman, W.G.B. 1954. Acetylated Sudan Black B as a more specific histochemical reagent for lipides. Qrtly. J. Microscopicalence 95:321-322.

Chua, L.S. 2016. Untargeted MS-based small metabolite identification from the plant leaves and stems of Impatiens balsamina. Plant Physiol. Biochem. 106:16-22.

Dahlqvist, A., I. Olsson, and A. Nordén. 1965. The periodate-Schiff reaction: Specificity, kinetics, and reaction products with pure substrates. J. Histochem. Cytochem. 13:423-430.

Franceschi, V.R. and H.T. Horner. 1980. Calcium oxalate crystals in plants. Bot. Rev. 46:361-427.

Franceschi, V.R. and P.A. Nakata. 2005. Calcium oxalate in plants: Formation and function. Annu. Rev. Plant Biol. 56:41-71.

Hu, S.Y. 1990. A cytochemical technique for demonstration sections of lipid drops, starch and protein bodies in thick resin sections. Acta Bot. Sin. 32:841-846.

Kang, S.N., Y.M. Goo, M.R. Yang, R.I. Ibrahim, J.H. Cho, I.S. Kim, and O.H. Lee. 2013. Antioxidant and antimicrobial activities of ethanol extract from the stem and leaf of Impatiens balsamina L. (Balsaminaceae) at different harvest times. Molecules 18:6356-6365. 
Lersten, N.R. and H.T. Horner. 2005. Development of the calcium oxalate crystal macropattern in pomegranate (Punica granatum, Punicaceae). Amer. J. Bot. 92:1935-1941.

Liu, C.W., K.M. Cong, Y.Y. Cai, and X. Zhen. 2006. Characteristics of calcium oxalate crystals of several species in Impatiens and their taxonomic significance. Life Sci. Res. 10:328-332.

McNair, J.B. 1932. The interrelation between substances in plants: Essential oils and resins, cyanogen and oxalate. Amer. J. Bot. 19:255-271.

Nakata, P.A. 2012. Engineering calcium oxalate crystal formation in Arabidopsis. Plant Cell Physiol. 53:1275-1282.

Nakata, P.A. 2015. An assessment of engineered calcium oxalate crystal formation on plant growth and development as a step toward evaluating its use to enhance plant defense. PLoS One 10:e0141982.

$\mathrm{Oku}, \mathrm{H}$. and K. Ishiguro. 2002. Cyclooxygenase-2 inhibitory 1,4naphthoquinones from Impatiens balsamina L. Biol. Pharm. Bul. 25:658-660.

Pacini, E. 1996. Types and meaning of pollen carbohydrate reserves. Sex. Plant Reprod. 9:362-366.

Sakunphueak, A., P. Tansakul, K. Umehara, H. Noguchi, and P. Panichayupakaranant. 2013. Effect of methionine on production of naphthoquinones in Impatiens balsamina root cultures and detection of some secondary metabolites. Pharm. Biol. 51:36-41.
Shin, J.A., M.H. Ryu, K.H. Kwon, B. Choi, and S.D. Cho. 2015. Down-regulation of Akt by methanol extracts of Impatiens balsamina L. promotes apoptosis in human oral squamous cell carcinoma cell lines. J. Oral Pathol. Med. 44:420-428.

Su, B.L., R. Zeng, J.Y. Chen, C.Y. Chen, J.H. Guo, and C.G. Huang. 2012. Antioxidant and antimicrobial properties of various solvent extracts from Impatiens balsamina L. stems. J. Food Sci. 77:C614C619.

Yang, S.J., C. Cheng, T.D. Song, and H.Q. Tian. 2013a. The character of anther development of Impatiens balsamina. Subtrop. Plant Sci. 42:283-287.

Yang, S.J., D.M. Wei, C. Cheng, T.D. Song, and H.Q. Tian. 2013b. Histochemical study of polysaccharides and lips on the developing anther of I. balsamina. Acta Botanica Boreal-Occident Sinica 33:1786-1791.

Yang, X., D.K. Summerhurst, S.F. Koval, C. Ficker, M.L. Smith, and M.A. Bernards. 2001. Isolation of an antimicrobial compound from Impatiens balsamina L. using bioassay-guided fractionation. Phytother. Res. 15:676-680.

Zhang, D., W. Liang, C. Yin, J. Zong, F. Gu, and D. Zhang. 2010. OsC6, encoding a lipid transfer protein, is required for postmeiotic anther development in rice. Plant Physiol. 154:149-162. 